\title{
Erratum: Flavor Constraints from Unitarity and Analyticity [Phys. Rev. Lett. 125, 081601 (2020)]
}

\author{
Grant N. Remmen and Nicholas L. Rodd॰
}

(Q) (Received 10 September 2021; published 28 September 2021)

DOI: 10.1103/PhysRevLett.127.149901

For the conventions adopted in our Letter, two-derivative four-fermion operators should generally be defined with an overall minus sign, in order to ensure the coefficients satisfy our positivity bounds as stated. In particular, all operators in Eq. (2) and (3), should be updated with an overall negative. As an explicit example, the first line of Eq. (2) should read

$$
\mathcal{O}_{1}[\psi]=-c_{m n p q}^{\psi, 1} \partial_{\mu} J_{\nu}[\psi]_{m n} \partial^{\mu} J^{\nu}[\psi]_{p q}, \quad \psi=\text { any }
$$

In the paragraph above Eq. (13), in order for the bound to be established on $a_{m n p q}^{d e, 1}$ and not $a_{m n p q}^{e d, 1}$, the sentence defining the scattered states should read "We start by scattering $d$ and $e$, so we take $\left|\psi_{2,3}\right\rangle$ as in Eq. (6), with $\gamma_{m}=\beta_{m}^{*}$, but take $\left|\psi_{1,4}\right\rangle$ to instead be $\alpha_{m i}\left|\bar{d}_{m}\right\rangle$ and $\alpha_{m i}^{*}\left|d_{m}\right\rangle$, respectively."

In the UV Completion section, the first paragraph describing the completion with $\phi_{m n}^{\mu \nu}$ should be removed, as the example is pathological. The massive spin-2 completion is not, however, the Wilson coefficients should be corrected to read

$$
\begin{aligned}
c_{m n p q}^{e, 1} & =\kappa^{2}\left(4 \delta_{m q} \delta_{n p}+\delta_{m n} \delta_{p q}\right) / 4 m^{2}, \\
c_{\alpha \beta}^{e, 1} & =\kappa^{2}\left(4|\alpha|^{2}|\beta|^{2}+|\alpha \cdot \beta|^{2}\right) / 4 m^{2},
\end{aligned}
$$

so that the second expression is now positive without the need to invoke the Cauchy-Schwarz inequality.

Published by the American Physical Society under the terms of the Creative Commons Attribution 4.0 International license. Further distribution of this work must maintain attribution to the author(s) and the published articles title, journal citation, and DOI. 